\title{
Ice nucleation forced by transient electric fields
}

\author{
Jens-Michael Löwe $\odot^{*}$ and Volker Hinrichsen \\ High-Voltage Laboratories, Technical University of Darmstadt, Darmstadt, 64283, Germany \\ Markus Schremb and Cameron Tropea $\odot$ \\ Institute of Fluid Mechanics and Aerodynamics, Technical University of Darmstadt, Darmstadt, 64283, Germany
}

(Received 8 April 2021; accepted 16 November 2021; published 2 December 2021)

\begin{abstract}
Icing affects many technical systems, like aircraft or high-voltage power transmission and distribution in cold regions. Ice accretion is often initiated by ice nucleation in sessile supercooled water droplets and is influenced by several influencing factors, of which the impact of electric fields on ice nucleation is still not completely understood. Especially the influence of transient electric fields is rarely or not at all investigated, even though it is of great interest, e.g., for high-voltage transmission lines or for the food industry. In the present study the impact of transient electric fields on ice nucleation in supercooled sessile water droplets is experimentally investigated under well-defined conditions. A set of droplets is cooled down to a certain temperature and is subsequently exposed to electric fields generated from standard lightning or standard switching impulse voltages, which are commonly used for testing of high-voltage equipment. The nucleation behavior of individual droplets is captured using a high-speed camera and the effect of the transient electric field on ice nucleation is analyzed by considering both the singular and the stochastic nature of nucleation. While the singular nature of nucleation is referred to during analysis of the relative number of droplets remaining liquid long times after the impulse voltage, its stochastic nature is accounted for in the analysis of the temporal evolution of the relative number of frozen droplets. It is shown that low electric field strengths $(\hat{E} \leqslant 6.52 \mathrm{kV} / \mathrm{cm})$ only have a negligible impact on ice nucleation, independent of the supercooling. In contrast, high electric field strengths $(\hat{E} \geqslant 9.78 \mathrm{kV} / \mathrm{cm})$ promote significantly ice nucleation. It is also shown that depending on the supercooling, the freezing delay of the different droplets in the ensemble may vary over several magnitudes for the same conditions. It is demonstrated that the electric field appears to indirectly affect the nucleation rate by generating droplet oscillations, finally promoting ice nucleation. The experiments clearly demonstrate the possibility to actively force ice nucleation by applying transient electric fields. These results improve the understanding of ice accretion on high-voltage insulators and may also lend insight into freezing processes in food industry. We expect that these results will be a valuable contribution in formulating and/or validating new nucleation models.
\end{abstract}

DOI: 10.1103/PhysRevE.104.064801

\section{INTRODUCTION}

Ice accretion is a severe problem for many applications like aircraft [1], ships [2,3], wind farms [4], food industry [5-7], or power engineering [8-10]. An accreted ice layer can lead to serious safety risks or may significantly decrease operational performance of the application. For aircraft or ships a safe operation is of first priority and ice accretion should be avoided, since the ice alters the shape of the wings or the ship, introduces additional loads and might lead to a functional failure by, for example, reducing the lift of the aircraft. In addition to the safety risk due to accreted ice, icing can cause a significant decrease of reliability as well as efficiency of a

\footnotetext{
*jens-michael.loewe@tu-darmstadt.de
}

Published by the American Physical Society under the terms of the Creative Commons Attribution 4.0 International license. Further distribution of this work must maintain attribution to the author(s) and the published article's title, journal citation, and DOI. system. Particularly in cold regions, high-voltage insulators and conductor ropes may be affected by ice accretion [9]. The insulators are specially designed for power transmission and distribution and should withstand different environmental conditions. The reliability of power transmission strongly depends on the performance of the insulators which insulate the high-voltage from ground potential [11,12]. Ice accretion alters the shape of the insulators by bridging the space between the weather sheds and may lead to increased creeping currents on the surface or to a flash-over caused by the reduced insulation distance [13,14]. Furthermore, the adhered ice leads to additional loads on the pylons or can cause line-galloping, which might lead to a collapse of the pylons [9]. Finally, the understanding of the physical mechanisms involved in ice accretion is essential for an optimization of the application and to ensure a reliable and safe operation.

Icing involves several physical mechanisms taking place on significantly different time and length scales. Whether icing occurs or not is primarily determined through nucleation, which initiates the freezing process. Ice nucleation depends on various influencing factors, like the supercooling of the liquid, 
foreign substances in contact with the liquid (ice nucleating particles) $[15,16]$, or other boundary conditions like varying pressure [17] or the presence of an electric field [18,19]. Although the effect of many influencing factors has been investigated in detail, the overall impact of electric fields on ice nucleation is still controversially discussed due to the fact that both a negligible and a large influence of the electric field has been shown in previous investigations [19-27]. Especially, the different boundary conditions in terms of type of the electric field, electric field strength, frequency and orientation of the electric field prohibit direct comparison and general conclusions.

As an example, these differences are demonstrated through a comparison of the results reported in Refs. [21,27]. While Stan et al. [21] investigated the influence of an electric field on homogeneous ice nucleation, our previous work examines the impact on heterogeneous ice nucleation [27]. The presence of a substrate significantly increases the nucleation temperature and may also alter the electric field distribution around the droplet, depending on the field orientation and the material properties. Hence, the electric field locally present at the threephase contact line might be significantly enhanced compared to a free floating droplet as used in Ref. [21]. In addition, the external electric field strength investigated in Ref. [27] is up to 4.4 times higher than in Stan et al. [21]. Even though the electric field is higher in Ref. [27], the droplets are less deformed because of the pinned contact line.

As revealed by Löwe et al. [27], each droplet is associated with a minimum electric field strength necessary to influence ice nucleation. This characteristic field strength might even be higher for the case of homogeneous compared to heterogeneous ice nucleation, so that it is not reached by the experiments presented in literature. Furthermore, the most important difference between both studies is the frequency of the electric field. High-frequency fields as used by Stan et al. [21] lead to the formation of an electric field inside the droplet. The droplet is assumed to be a dielectric, because the charge relaxation time of water is much higher compared to the characteristic time of the electric field. Lowering the frequency of the electric field causes a change of the physical mechanism, because in this case the charge relaxation time of water is much shorter than the characteristic time of the electric field. Consequently, the droplet is assumed to be a perfect conductor, and thus no electric field is present in the droplet. Nevertheless, the external electric field still exerts an influence on ice nucleation. In addition, the influence of the electric field might be small compared to other influences and might be masked by more dominant factors like the stochastic nature of ice nucleation itself [28] or by variations caused by impurities in the sample. In summary, the different experimental conditions induce different physical mechanisms, and thus complicate the comparison of different experimental results.

Although the influence of electric fields has been controversially discussed in previous studies, molecular dynamics simulations have revealed an unequivocal impact of electric fields on ice nucleation [29-34]. However, in these studies the investigated electric field strengths are much higher than achievable in experiments. Nevertheless, Yan et al. [32] assume that localized high electric fields and the associated orientation of nearby water-dipoles might cause an increase of the local supercooling, finally resulting in the formation of a sufficiently large ice nucleus to induce heterogeneous ice nucleation. This mechanism might also be present in the present experiments, however, the type of electric field is also different, so a direct comparison is difficult.

Although the influence of constant and alternating electric fields was extensively investigated in the past using experiments and simulations, the influence of transient electric fields has been only rarely studied [35,36]. In most cases, alternating electric fields are assumed to be quasi-static and thus, are not considered as transient electric fields even if the field strength varies over time [37]. In contrast, transient electric fields are applied only for a short time (in the micro- and milliseconds range). Generally, these electric fields are characterised by a steep increase and a slower decrease of the electric field strength. Such transient electric fields might occur in power transmission and distribution systems due to lightning strikes in an overhead power line or during switching operations [38]. Within the scope of ice accretion, the abrupt change of the boundary conditions might serve as a disturbance of the metastable state of sessile supercooled droplets and therefore, a candidate for promoting ice nucleation.

In the present work, the impact of transient electric fields on ice nucleation in supercooled sessile water droplets is experimentally studied under well-defined conditions. A set of 40 sessile droplets is cooled down to a constant temperature between $\vartheta=-5.9^{\circ} \mathrm{C}$ and $\vartheta=-21^{\circ} \mathrm{C}$, and is subsequently exposed to standard lightning or switching impulse voltages with an electric field strength up to $\hat{E}=22.82 \mathrm{kV} / \mathrm{cm}$. For each impulse type, the same set of droplets is used for all field strengths to guarantee a high repeatability of the experiments by minimizing the influence of varying impurities inside the droplets. The experiments are repeated at least four times for each experimental condition, resulting in a minimum of 95 nucleation events for almost all conditions; only for high switching impulse voltages of $\hat{E}=19.56 \mathrm{kV} / \mathrm{cm}$ is the number of nucleation events varied between 35 and 88 due to significant droplet disintegration during the impulse voltage application. A high-speed camera is used to capture the nucleation events and to investigate ice nucleation with respect to different timescales.

\section{EXPERIMENTAL SETUP}

The experiments are performed using SAPPHIRE, which has been presented in detail in Ref. [39] and is therefore only briefly described here. A schematic of the experimental setup is shown in Fig. 1. Up to 40 water droplets with a mean diameter of the wetted surface between $d=0.96 \mathrm{~mm}$ and $d=1.07 \mathrm{~mm}$ are placed on a specimen holder. The droplets are generated using a piezo driven drop-on-demand generator built according to Harris et al. [40] and using high purity water (Millipore Milli-Q Type 1, electrical conductivity of $\gamma_{\mathrm{el}}=$ $5.5 \times 10^{-6} \mathrm{~S} / \mathrm{m}$ at $25^{\circ} \mathrm{C}$ ). The droplets are deposited on a sapphire glass sheet of high heat conductivity, which serves as the sample holder. Prior to droplet deposition, the glass sheet is carefully cleaned by rinsing isopropanol over the substrate. After deposition, the glass sheet with the droplets is placed inside a groove in the ceramic body. Subsequently, the glass sheet and the droplets on top are covered with silicone oil to 


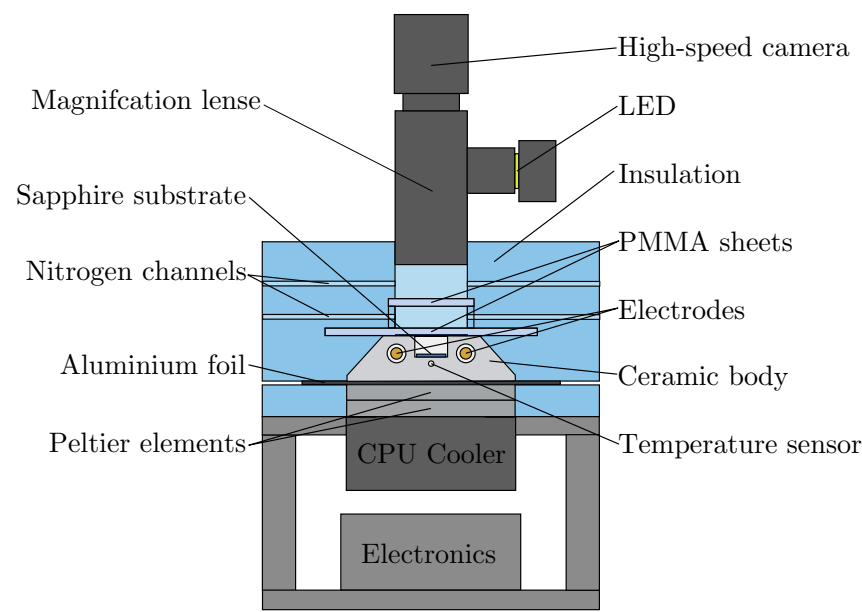

FIG. 1. Schematic of the experimental setup used in the present study. Adapted and published with permission from Ref. [39] under the Creative Common License-Attribution 4.0 International (CC BY 4.0).

prevent droplet evaporation, condensation of additional water and to reduce the Wegener-Bergeron-Findeisen process [41].

The ceramic body comprises two electrodes embedded in conductive adhesive to generate an electric field tangentially aligned to the sapphire substrate. The ceramic body defines the position of the droplets inside the electric field and ensures good heat distribution in the substrate because of the high heat conductivity of $40 \mathrm{~W} / \mathrm{m} \mathrm{K}$ at $25^{\circ} \mathrm{C}$ and $80 \mathrm{~W} / \mathrm{m} \mathrm{K}$ at $-40^{\circ} \mathrm{C}$. Two stacked Peltier elements controlled by a commercially available controller are used to cool down the ceramic block. The heat generated by and transferred through the Peltier elements is dissipated using a water based CPU cooler. The actual temperature of the droplets cannot be measured directly because the temperature sensor might influence the electric field distribution or ice nucleation itself. Hence, the temperature is measured inside the ceramic block and is correlated to the current droplet temperature via calibration. A more detailed description of the calibration procedure can be found in Löwe et al. [39]. In addition to the calibration for a constant cooling rate, reported in Löwe et al. [39], the indirect temperature measurement is also calibrated for the present experiments performed at constant temperature. To minimize heat exchange with the surrounding and to ensure well-defined conditions, the entire setup is housed in Styrofoam and placed inside a climate chamber operated at a constant temperature of $\vartheta=7.5^{\circ} \mathrm{C}$. Ice nucleation in the droplets is observed from above using a high-speed (Photron Fastcam MC2.1-10K) video camera. A magnification lens (Navitar 12x Zoom Lens) with coaxial illumination is attached to the camera to capture the nucleation process with a spatial resolution of approximately $2.7 \times 10^{-5} \mathrm{~m} / \mathrm{px}$. The video data is analyzed using an in-house Matlab code to detect the nucleation of the individual droplets by the vanishing glare points as shown in Löwe et al. [39] and to determine the temporal evolution of the frozen fraction of droplets during and after the high voltage impulse.

The electric field is generated using two single-stage impulse generators [38] with a maximum voltage level of $|U|=$
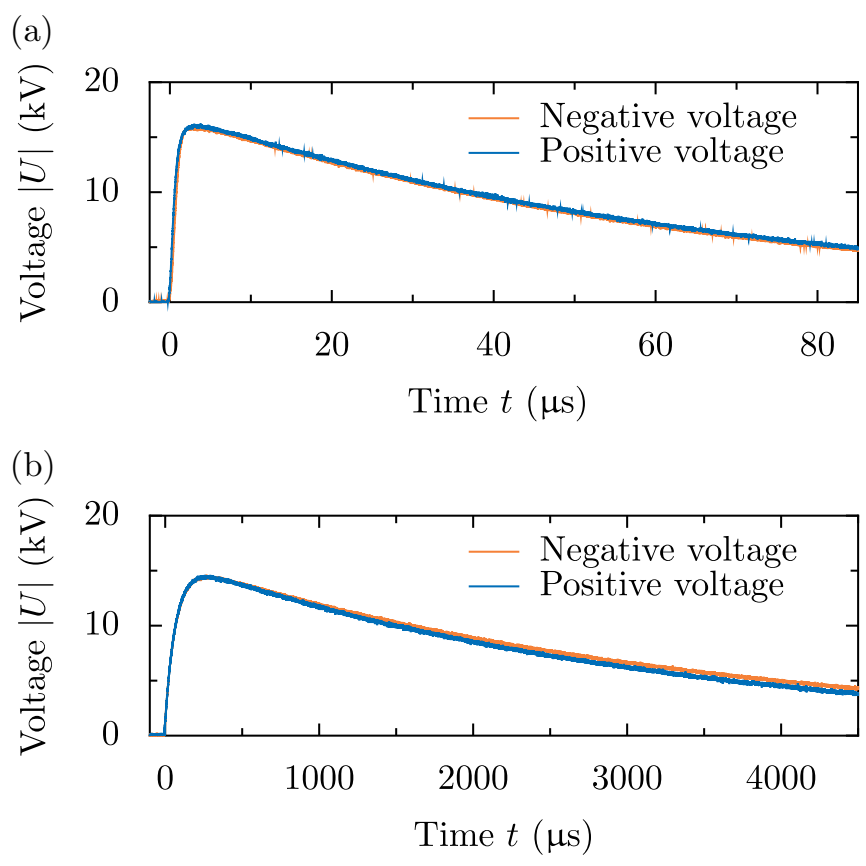

FIG. 2. Example measurements of the temporal evolution of the voltage generated by the impulse generator for (a) standard lightning impulse voltage $(1.2 / 50 \mu \mathrm{s})$ and (b) standard switching impulse voltage $(250 / 2500 \mu \mathrm{s})$. Each curve shows the norm of the supplied voltage signal of one electrode.

$25 \mathrm{kV}$ for each of the generators. Each impulse generator is connected to one of the electrodes in the ceramic and is operated with opposite polarity to generate an electric field tangentially aligned to the sapphire substrate, which is not affected by the grounded protection layer below the ceramic body. A self-made Thyristor switch is used to trigger both impulse generators at the same time. Example measurements of the temporal evolution of the applied voltage during a standard lightning impulse voltage $(1.2 / 50 \mu \mathrm{s})$ and a standard switching impulse voltage (250/2500 $\mu$ s) are shown in Fig. 2. Both signals are triggered simultaneously, and their evolution is in excellent agreement with respect to the maximum amplitude and evolution of the signal, finally resulting in a well-aligned electric field for the deposited droplets. Due to the different characteristic times of the impulses, they cover completely different timescales, which enables to not only study the general effect of a highly transient high voltage impulse on ice nucleation, but also to examine the role of time.

\section{EXPERIMENTAL PROCEDURE}

The sessile droplets are cooled down to a constant temperature between $\vartheta=-5.9^{\circ} \mathrm{C}$ and $\vartheta=-21^{\circ} \mathrm{C}$ with a constant cooling rate of $\dot{T}=5 \mathrm{~K} / \mathrm{min}$. Subsequently, the temperature is held constant for at least 2 min to ensure a constant temperature of the droplets before the electric field is applied. Droplets that are already frozen before the electric field is applied, are not taken into account during the analysis. Recording of the camera is triggered simultaneously with the electric field, so that the video data is automatically synchronized to the transient electric field. The observation time is lim- 
ited by the maximum recording time of the camera and is constant for all experiments at $\Delta t=1.5 \mathrm{~s}$ with a frame rate of $4000 \mathrm{fps}$. Consequently, the experimental results for the final liquid and frozen fraction of the droplets are compared at $t=$ $1.5 \mathrm{~s}$ after the electric field is applied. Note that droplets freezing later than $t=1.5 \mathrm{~s}$ are not taken into account in the present analysis. Although individual droplets might also freeze after longer freezing delays, only those nucleation events observed for $t<1.5 \mathrm{~s}$ are assumed to be possibly related to the transient electric field. However, such large freezing delays in the order of several seconds were only rarely observed in the present study.

After an experiment, the droplet ensemble is heated up to a temperature of about $\vartheta \approx 5^{\circ} \mathrm{C}$ to melt the frozen droplets and to prepare the droplet ensemble for the next experiment. Each experimental condition defined by the degree of supercooling and an electric field strength is repeated at least four times to generate a sufficient data basis. Due to the fact that the droplet ensemble is essentially the same for all experiments performed with one of the two impulse types, external influences like a variation of the impurities inside the droplets can be neglected. During the analysis only droplets with a diameter within a $\pm 10 \%$ tolerance around the mean value of the droplet diameters of all considered droplets are accounted for.

\section{RESULTS}

Different influencing factors such as the type of the electric field, the electric field strength or its frequency were identified to have an impact on ice nucleation [27], indicating that not only the characteristics of the electric field (such as electric field strength or frequency), but also time (e.g., duration of application) plays an important role in the scope of electro-freezing. Therefore, it is expected that the electric field strength as well as the type of the impulse voltage and thus, the shape, including the steepness and the duration of the impulse, have an impact on ice nucleation.

\section{A. Standard lightning impulse voltage $(1.2 / 50 \mu s)$}

According to IEC 60060-1 the standard lightning impulse voltage $(1.2 / 50 \mu \mathrm{s})$ is defined by a front time of $1.2 \mu \mathrm{s}$ and a time to half-value of $50 \mu$ s [see Fig. 2(a) for an example], which leads to a steep increase and a much slower decrease of the voltage. In the following the standard lightning impulse voltage $(1.2 / 50 \mu \mathrm{s})$ is simply referred to as a lightning impulse voltage. The characteristic times of the impulse voltage are significantly shorter than the typical timescale of droplet oscillations, which are usually in the order of tens to hundred milliseconds for alternating electric fields [42]. Consequently, the underlying physical mechanism for the impact of lightning impulse voltages on ice nucleation can be expected to be different from the impact of constant or alternating electric fields, whose characteristic times are much more comparable to the characteristic time of droplet oscillation.

The final frozen fraction $N_{\mathrm{f}} / N_{0}$ determined a long time $(t>$ $1 \mathrm{~s})$ after applying the lightning impulse voltage, depending on the ensemble supercooling and the electric field strength $\hat{E}$, is shown in Fig. 3. Here, the electric field strength $\hat{E}$ corre-

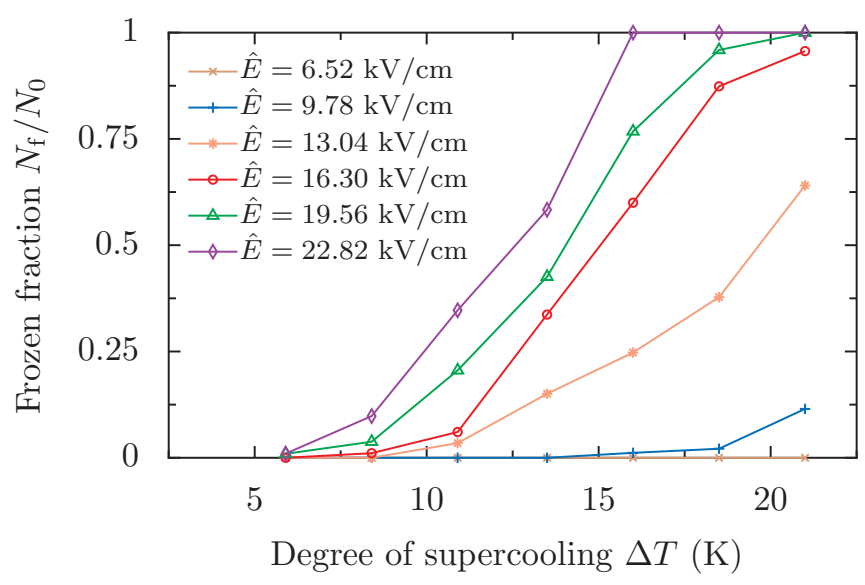

FIG. 3. Final frozen fraction $N_{\mathrm{f}} / N_{0}$ measured long times after applying the impulse voltage, depending on the ensemble supercooling $\Delta T$ for lightning impulse voltages of varying peak electric field strength $\hat{E}$.

sponds to the peak value of the transient electric field strength and therefore, it is the highest field strength the droplets are exposed to during the transient increase and decay of the electric field. Note that the data presented in this figure are captured with a low-speed camera (Basler A631fc). Due to the short duration of the physical process $(\Delta t<1.5 \mathrm{~s})$, all remaining experiments are investigated with the high-speed camera to achieve a higher temporal resolution without losing any information caused by the limited record duration of the camera. The frozen fraction $N_{\mathrm{f}} / N_{0}$ is defined as the ratio of actually frozen droplets $N_{\mathrm{f}}$ and the number of droplets $N_{0}$, which are taken into account for the analysis. For the experiments performed with lightning impulse voltages a minimum of 95 nucleation events is observed for each combination of droplet temperature and electric field strength.

As shown in the figure, for a low electric field strength, $\hat{E}=6.52 \mathrm{kV} / \mathrm{cm}$, the electric field has no influence on ice nucleation, independent of the degree of supercooling. None of the droplets is forced to freeze by the transient electric field. However, increasing the electric field strength leads to an increase of the frozen fraction, depending on the degree of supercooling. The higher the electric field strength the higher is the frozen fraction for a given supercooling. For electric field strengths of $\hat{E} \leqslant 16.30 \mathrm{kV} / \mathrm{cm}$, even the highest supercooling of $\Delta T=21 \mathrm{~K}$ does not cause $N_{\mathrm{f}} / N_{0}=1$, i.e., some droplets remain liquid. For a further increase of the electric field strength also the frozen fraction increases for a constant supercooling and always results in a fully frozen ensemble for a supercooling of $\Delta T=21 \mathrm{~K}$. Moreover, the highest electric field strength results in a completely frozen ensemble even at lower supercooling. For the highest electric field strength of $\hat{E}=22.82 \mathrm{kV} / \mathrm{cm}$ a frozen fraction of $N_{\mathrm{f}} / N_{0}=1$ is already observed for a supercooling of $\Delta T=16 \mathrm{~K}$. In addition, the mean freezing temperature $\vartheta_{0.5}$, corresponding to $N_{\mathrm{f}} / N_{0}=$ 0.5 , decreases with increasing electric field strength. The approximate values for the mean freezing temperature $\vartheta_{0.5}$ obtained from linear interpolation are shown in Table I for varying electric field strength. 
TABLE I. Approximated mean nucleation temperature $\vartheta_{0.5}$ depending on the electric field strength $\hat{E}$ for lightning impulse voltage. The mean nucleation temperature corresponds to $N_{\mathrm{f}} / N_{0}=0.5$ and characterizes the approximate temperature, at which half of the ensemble freezes for a given electric field strength.

\begin{tabular}{ll}
\hline$\hat{E}$ in $\mathrm{kV} / \mathrm{cm}$ & $\vartheta_{0.5}$ in ${ }^{\circ} \mathrm{C}$ \\
\hline 13.04 & -19.66 \\
16.30 & -15.05 \\
19.56 & -14.04 \\
22.82 & -12.58 \\
\hline \hline
\end{tabular}

To examine the effect of the droplet ensemble and its associated nucleation site spectrum on ice nucleation, some experiments with a lightning impulse voltage were repeated. A fresh droplet ensemble with an almost identical mean droplet diameter $(d=1.07 \mathrm{~mm}$ compared to $d=0.96 \mathrm{~mm}$ in the previous ensemble) is exposed to lightning impulse voltages with the same electric field strength as before. A comparison of the dependence of the frozen fraction of droplets on supercooling and electric field strength observed for the different droplet ensembles is shown in Fig. 4. The data shown by hollow symbols and solid lines correspond to the data already shown in Fig. 3. In comparison, the results from the new ensemble are shown by the filled symbols and the dashed lines. Identical colors indicate the same electric field strength for both ensembles.

As shown in the figure, the data from both ensembles are in very good agreement. For the lowest electric field strength of $\hat{E}=6.52 \mathrm{kV} / \mathrm{cm}$ almost no deviation between the individual sets is observable; the electric field does not affect ice nucleation regardless of supercooling. Higher electric field strengths, using a fresh droplet ensemble, are associated with a slight decrease of the frozen fraction for $\Delta T \geqslant 10 \mathrm{~K}$. However, ice nucleation, i.e., the dependence of $N_{\mathrm{f}} / N_{0}$ on supercooling and the electric field strength, is very comparable

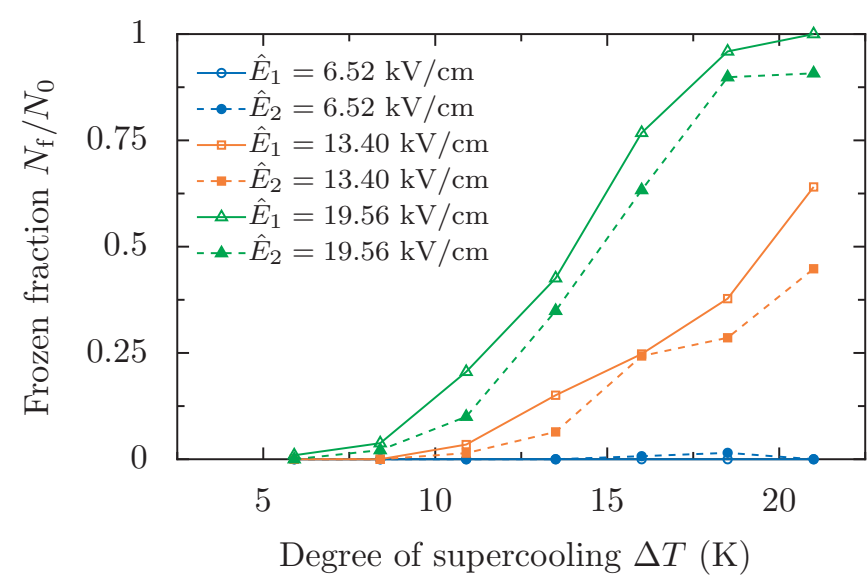

FIG. 4. Frozen fraction $N_{\mathrm{f}} / N_{0}$ for two different droplet ensembles depending on supercooling $\Delta T$ and the electric field strength $\hat{E}$. Both ensembles are exposed to standard lightning impulse voltages. The data already shown in Fig. 3 is indicated by subscript 1 $(d=0.96 \mathrm{~mm})$ and is compared to the data obtained with a fresh ensemble (subscript 2, $d=1.07 \mathrm{~mm}$ ).

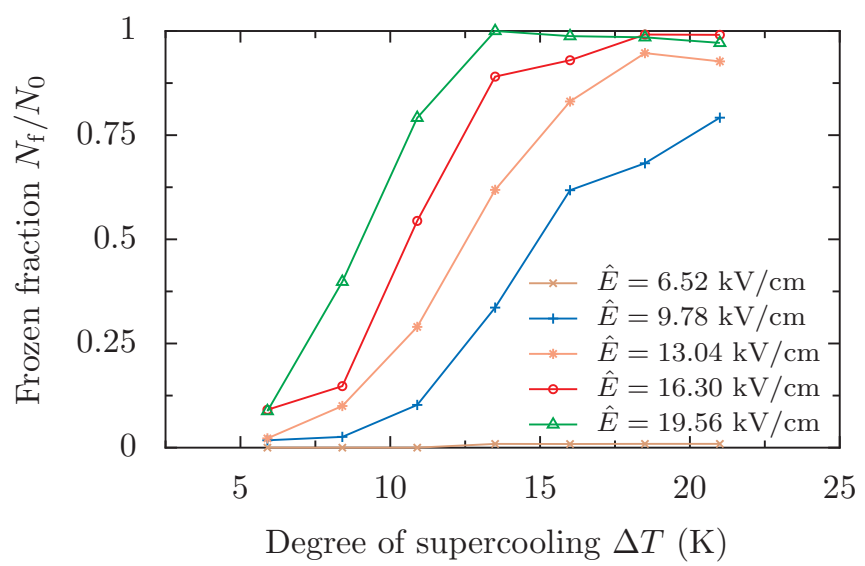

FIG. 5. Frozen fraction $N_{\mathrm{f}} / N_{0}$ depending on the supercooling $\Delta T$ as well as the electric field strength $\hat{E}$ for a switching impulse voltage.

for the different droplet ensembles, demonstrating the high repeatability of the experiments and the significance of the effect of the electric field on ice nucleation.

The slight deviation between the data observable in Fig. 4 presumably originates from the changed spectrum of nucleation sites and the slightly different mean droplet sizes in the different droplet ensembles. Even a careful sample preparation and producing the droplets from the same bulk liquid cannot completely rule out the influence of impurities in the water [43]. Nevertheless, the data is in very good agreement and therefore, a transient high voltage field is proven to significantly affect ice nucleation in sessile water droplets.

\section{B. Standard switching impulse voltage $(250 / 2500 \mu \mathrm{s})$}

In comparison to the standard lightning impulse voltage $(1.2 / 50 \mu \mathrm{s})$, the typical timescales associated with a standard switching impulse voltage $(250 / 2500 \mu \mathrm{s})$ are two orders of magnitude longer and consequently, the corresponding temporal gradients of the field strength are smaller. The standard switching impulse is characterized by a time to peak of $250 \mu \mathrm{s}$ and a time to half-value of $2500 \mu$ s [see Fig. 2(b) for an example, IEC 60060-1]. Therefore, the electric field resulting from a standard switching impulse is applied for a significantly longer time duration compared to a standard lightning impulse voltage, which provides insight into the mechanisms taking place during ice nucleation in a highly transient electric field. The standard switching impulse voltage $(250 / 2500 \mu \mathrm{s})$ is referred to as switching impulse voltage. Similar to the case of lightning impulse voltages, the characteristic timescales of the impulse are significantly smaller than the typical resonance frequency of droplets under the impact of alternating electric fields.

The final frozen fraction of droplets for the experiments with switching impulse voltages applied is shown in Fig. 5. For the experiments performed with switching impulse voltages a minimum of 95 nucleation events is observed for an electric field strength $\hat{E}<19.56 \mathrm{kV} / \mathrm{cm}$, whereas a minimum of 35 nucleation events is analyzed for $\hat{E}=19.56 \mathrm{kV} / \mathrm{cm}$. Especially for an electric field strength of $\hat{E}=19.56 \mathrm{kV} / \mathrm{cm}$ and large supercooling, the number of droplets taken into 
TABLE II. Approximated mean nucleation temperature $\vartheta_{0.5}$ depending on the electric field strength $\hat{E}$ for switching impulse voltage. The mean nucleation temperature corresponds to $N_{\mathrm{f}} / N_{0}=$ 0.5 and characterizes the approximate temperature at which half of the ensemble freezes for a given electric field strength.

\begin{tabular}{cc}
\hline \hline$\hat{E}$ in $\mathrm{kV} / \mathrm{cm}$ & $\vartheta_{0.5}$ in ${ }^{\circ} \mathrm{C}$ \\
\hline 9.78 & -14.95 \\
13.04 & -12.56 \\
16.30 & -10.62 \\
19.56 & -9.05 \\
\hline \hline
\end{tabular}

account during the analysis is significantly reduced due to the release of tiny droplets from the main droplets for higher field strengths, which will be elaborated below. The various repetitions of the experiments and the release of tiny droplets causes a continuous decrease of the droplet volumes: these drops are excluded from further analysis. However, the remaining data basis still allows statistically significant conclusions concerning the effect of a transient electric field on ice nucleation.

Similar to the experiments with lightning impulse voltages, the lowest electric field strength of $\hat{E}=6.52 \mathrm{kV} / \mathrm{cm}$ has no significant impact on ice nucleation in the sessile droplets, independent of the degree of supercooling. The frozen fraction increases with both increasing supercooling and increasing field strength. However, in contrast to the lightning impulse voltage, the effect of the switching impulse voltage on ice nucleation is significantly stronger for the same electric field strength. While a lightning impulse voltage with $\hat{E}=9.78 \mathrm{kV} / \mathrm{cm}$ does not significantly affect ice nucleation for lower supercooling and results in a small frozen fraction of only $12.5 \%$ at $\Delta T=21 \mathrm{~K}$ (see Fig. 3), approximately $80 \%$ of the droplets are frozen at $\Delta T=21 \mathrm{~K}$ after applying a switching impulse voltage, as shown in Fig. 5. A switching impulse also significantly enhances nucleation for smaller supercooling at that low field strength. It enhances ice nucleation such that a frozen fraction of $12.5 \%$ is observed already for a smaller supercooling of $\Delta T=10.9 \mathrm{~K}$. Moreover, a fully frozen droplet ensemble, $N_{\mathrm{f}} / N_{0}=1$, is observed already for an electric field strength of $\hat{E}=16.30 \mathrm{kV} / \mathrm{cm}$ at $\Delta T=21 \mathrm{~K}$.

For the highest electric field strength of $\hat{E}=19.56 \mathrm{kV} / \mathrm{cm}$, the droplet supercooling associated with $N_{\mathrm{f}} / N_{0}=1$ is significantly decreased. While that electric field strength results in $N_{\mathrm{f}} / N_{0}=1$ only for $\Delta T=21 \mathrm{~K}$, in the case of a lightning impulse the switching impulse causes $N_{\mathrm{f}} / N_{0}=1$ even for a droplet supercooling of only $\Delta T=13.5 \mathrm{~K}$. Finally, a switching impulse voltage with a high electric field strength causes $N_{\mathrm{f}} / N_{0}>0$ even for the smallest supercooling of $\Delta T=5.9 \mathrm{~K}$. Similar to lightning impulse voltages, the mean nucleation temperature decreases with increasing electric field strength. The mean nucleation temperatures are generally lower in the case of a switching impulse voltage compared to a lightning impulse voltage. Table II shows the approximate mean nucleation temperatures for the data shown in Fig. 5.

The impact of switching impulse voltages on ice nucleation is observed to be significantly higher compared to the effect of lightning impulse voltages. Thus, typical timescales associated with the switching impulse voltage result in a much (a)

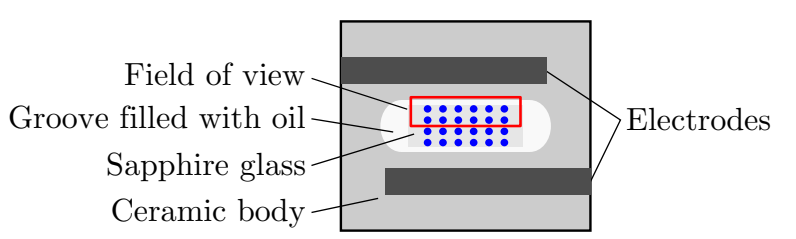

(b)

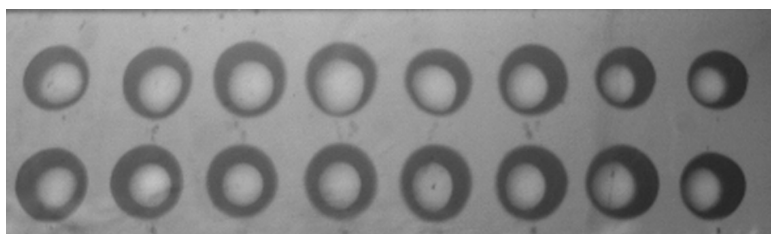

(c)

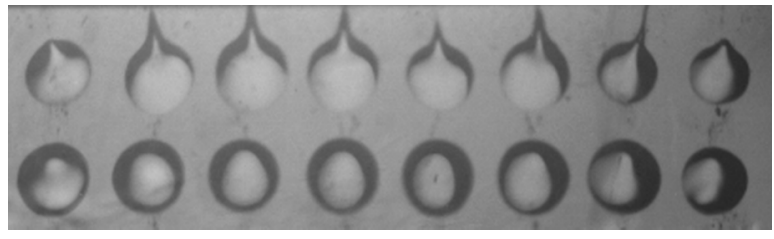

(d)

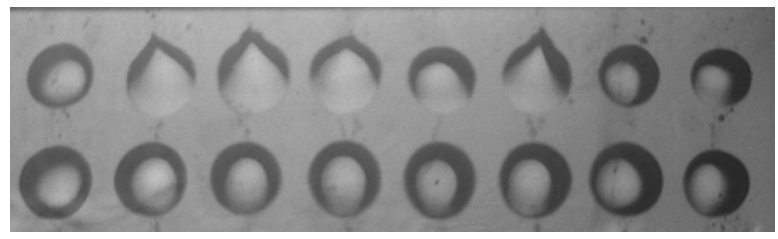

(e)

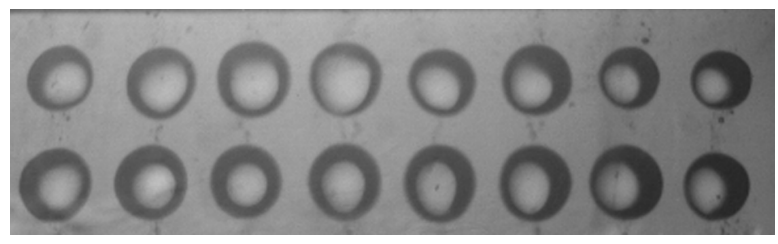

FIG. 6. Part of a droplet ensemble during the impact of a switching impulse voltage with $\hat{E}=19.56 \mathrm{kV} / \mathrm{cm}$ at $\vartheta=-5.9^{\circ} \mathrm{C}$ (no freezing is observed) in top view. Each image shows the droplets to a different time after triggering of the impulse. (a) Sketch of arrangement, (b) Time of trigger, (c) deformation of the droplets after $t=2 \mathrm{~ms}$, (d) after $t=4 \mathrm{~ms}$, and (e) after $t=6 \mathrm{~ms}$. The mean droplet diameter is $d=1.01 \mathrm{~mm}$.

longer duration to which the droplet ensemble is exposed at high electric field conditions compared to the case of the lightning impulse voltage. Consequently, different mechanisms potentially influential for ice nucleation may be affected by this enhanced duration of the electric field. On the microscopic level, water molecules have more time to better rearrange, and on the macroscopic level viscous and inertial forces are more likely to be overcome than in the case of a shorter impulse duration. Particularly, the latter is associated with a significantly increased droplet deformation compared to the situation during the lightning impulse.

A time series of a droplet ensemble, illustrating the impact of a switching impulse voltage on droplet deformation in top view, is shown in Fig. 6. The droplets are cooled down and held at a temperature of $\vartheta=-5.9^{\circ} \mathrm{C}$ for $5 \mathrm{~min}$ before triggering an impulse with $\hat{E}=19.56 \mathrm{kV} / \mathrm{cm}$ at $t=0 \mathrm{~ms}$. As shown in Figs. 6(c) and 6(d), corresponding to 2 and $4 \mathrm{~ms}$ after applying the impulse voltage, the droplets are significantly deformed through the transient electric field. Some droplets 

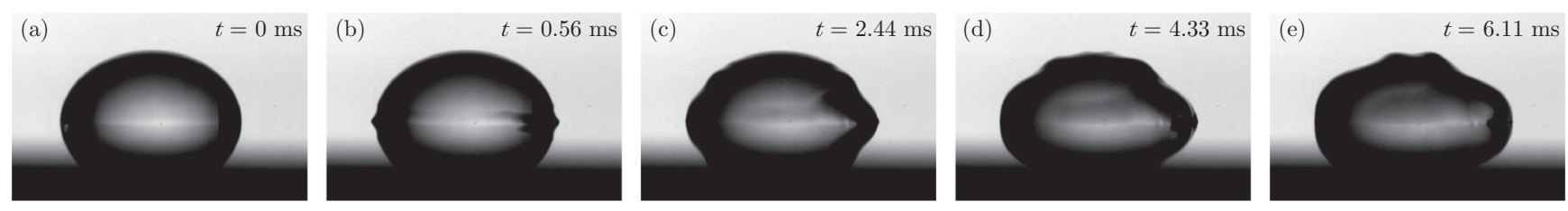

FIG. 7. Side view time series of a sessile water droplet $(V=50 \mu l)$ surrounded by air and exposed to a switching impulse voltage $(\hat{E}=$ $18.43 \mathrm{kV} / \mathrm{cm}$ ) under ambient conditions. The corresponding video can be found in the Supplemental Material [47].

located close to the electrodes (top row in the pictures) are significantly deformed. These droplets form a Taylor cone parallel to the substrate, which is associated with the release of tiny droplets from the deformed droplets under observation, as reported in Refs. [44,45]. Note that also droplets farther away from the electrodes sometimes release tiny droplets, which can be observed in the space in-between the main droplets. Due to the fact that the released droplets are rather small, the change of the droplet volume is almost negligible during a single experiment. However, performing several repetitions with the same set of droplets will eventually lead to a noticeable volume decrease for individual droplets.

While the largest deformation of the droplets is shown in Fig. 6(c), a retreating of the deformation is shown in Fig. 6(d). After a time of $t=6 \mathrm{~ms}$ the droplets are in their initial state. Interestingly, even if the droplets are significantly deformed during the applied impulse voltage, the wetted surface area and the shape of each individual droplet is not permanently altered. The appearance of the droplets is almost the same before and after application of the impulse voltage. The contact line of the droplet slightly moves during the impact of the electric field but returns to almost the same position in which it was prior to application of the electric field.

In contrast to lightning impulse voltages, the switching impulse voltage significantly deforms the droplets. For lightning impulse voltages the movement of the contact line is much smaller and no formation of a Taylor cone is observable. Only small oscillations can be recognized. Droplet coalescence is prevented by the rather large droplet-droplet separation, although in general the deformation of the droplets can lead to coalescence of neighboring droplets [46]. The top-view of the droplets allows observation of the droplet deformation and nucleation.

A side view image sequence illustrating the macroscopic behavior of a water droplet exposed to a switching impulse voltage is shown in Fig. 7. The corresponding video can be found in the Supplemental Material [47]. The applied electric field leads to a force which is concentrated at the three-phase contact line. As soon as the electric field is applied, the threephase contact line is forced outwards. These disturbances form capillary waves traveling from both sides of the droplet towards the center, see Figs. 7(b) and 7(c). As soon as these waves reach the top of the droplet they interact with each other and lead to further droplet oscillations, as shown in Figs. 7(d) and 7(e). After the initial impulse and motion of the contact line, the contact line is pinned, while the droplet oscillates. Due to the liquid viscosity and surface tension the droplet oscillation decays over time. As soon as the electric field vanishes and the oscillation is completely damped, the threephase contact line moves towards its initial position. Whether the three-phase contact line returns to the initial position or not mainly depends on the electric field strength applied to the droplet and the local wetting properties of the substrate.

The droplet behavior under the impact of lightning impulse voltages is basically similar to the behavior during switching impulse voltages. However, the outward motion of the contact line is significantly smaller and hardly recognizable. Consequently, the disturbances initiated at the three-phase contact line are also much smaller and do not lead to significant droplet oscillations. In fact, the amplitude of the oscillation is much smaller and thus, the oscillations decay much faster compared to switching impulse voltages. The contact line immediately returns to the initial position after causing the disturbances.

Generally, the behavior is very similar to the behavior during switching impulse voltages, but the impact is almost negligible for a lightning impulse voltage. Both, Figs. 6 and 7 show that a transient impulse can have a significant impact on droplet motion and can result in large deformations and oscillations. However, the actual droplet deformation clearly depends on the type of the impulse and its corresponding timescales. The larger timescales of switching compared to lightning impulse voltages appear to be much more comparable to the typical timescale of droplet motion, thus resulting in more pronounced droplet deformation for the same electric field strength.

The electrical timescale characterizes the behavior of the liquid with respect to a perfect conductor or a leaky-dielectric and is given by $\tau_{\mathrm{el}}=\varepsilon / \gamma_{\mathrm{el}}$, where $\varepsilon=\varepsilon_{0} \varepsilon_{\mathrm{r}}$ is the permittivity of water defined through the vacuum permittivity, $\varepsilon_{0}$, and the relative permittivity, $\varepsilon_{\mathrm{r}}$, and $\gamma_{\mathrm{el}}$ is the electric conductivity of the water. Assuming a relative permittivity of $\varepsilon_{\mathrm{r}}=81$ yields a timescale of $\tau_{\mathrm{el}} \approx 10^{-4} \mathrm{~s}$ for water. Due to the fact that $\tau_{\mathrm{el}} / \tau_{\mathrm{E}} \ll 1$, the liquid can be assumed to be a perfect conductor when considering slow processes (e.g., exposition to power-frequency alternating voltages or DC voltages with $\tau_{\mathrm{E}}$ as a characteristic timescale of the electric field). An impulse voltage can be characterized by two different times; the front time and the time to half-value. Table III shows the characteristic timescales for both lightning and switching impulse voltages. A comparison of these timescales reveals that the timescale for a lightning impulse voltage is much smaller than the electric timescale of water, $\left(\tau_{1, \mathrm{LI}} \ll \tau_{\text {el }}\right.$ and $\left.\tau_{2, \mathrm{LI}} \ll \tau_{\mathrm{el}}\right)$. Hence, the liquid behaves as a dielectric during the impact of the lightning impulse voltage. As a consequence, the electric field inside the droplet does not vanish, which leads to electrical stresses normally and tangentially aligned to the droplet interface. In contrast, a droplet exposed to a switching impulse voltage behaves differently. Comparing the characteristic timescales of switching impulse voltage with 
TABLE III. Characteristic timescales for water (charge relaxation time) and time constants of the test voltages according to IEC 60060-1 and Ref. [38].

\begin{tabular}{lc}
\hline \hline Description & Characteristic timescale \\
\hline Charge relaxation time & $\tau_{\mathrm{el}}=\frac{\varepsilon}{\gamma_{\mathrm{el}}} \approx 10^{-4} \mathrm{~s}$ \\
Time to peak $(\mathrm{LI})$ & $\tau_{1, \mathrm{LI}} \approx 10^{-7} \mathrm{~s}$ \\
Time to half value $(\mathrm{LI})$ & $\tau_{2, \mathrm{LI}} \approx 10^{-5} \mathrm{~s}$ \\
Time to peak $(\mathrm{SI})$ & $\tau_{1, \mathrm{SI}} \approx 10^{-5} \mathrm{~s}$ \\
Time to half value $(\mathrm{SI})$ & $\tau_{2, \mathrm{SI}} \approx 10^{-3} \mathrm{~s}$ \\
\hline \hline
\end{tabular}

the characteristic electric timescale of water reveals that the droplet can be assumed as a dielectric during the time to peak, because $\tau_{1, \mathrm{SI}}<\tau_{\mathrm{el}}$. However, during the decrease of the electric field strength $\tau_{2, \mathrm{SI}}>\tau_{\mathrm{el}}$, so that the droplet can be assumed to be a perfect conductor. As a consequence, the electric field inside the droplet vanishes and there is no force tangentially aligned to the surface of the droplet generated by the electric field.

The electrical stress tangentially aligned to the droplet interface is a necessary requirement for the emission of small droplets from a Taylor cone [48]. However, only during switching impulse voltages is the characteristic time of the impulse decrease long enough to generate Taylor cones and consequently, the formation of a Taylor cone is only observed during switching impulse voltages.

The stronger impact of the switching impulse voltage on the droplet motion might also be the reason for its stronger impact on ice nucleation compared to lightning impulse voltages. The reorientation of the water molecules is much faster than the applied impulse voltages, because water molecules can be aligned by electric fields even for a frequency in the $\mathrm{GHz}$ range [49]. Hence, the difference between the lightning and switching impulse voltage presumably does not result from an effect on the molecular length scale, but may be based on the macroscopic motion of a droplet. Generally, the macroscopic oscillation of sessile droplets has a characteristic timescale in the order of milliseconds and thus, is significantly larger than the characteristic time of the impulse voltages. However, the longer characteristic times of the switching impulse cause significantly stronger droplet oscillations compared to a lightning impulse at the same field strength value. Therefore, it is assumed that the increased macroscopic droplet motion for longer characteristic times of the electric field is the main reason for the increased impact. This assumption will be analyzed in more detail in the following section.

\section{Singular versus stochastic behavior}

So far, the experimental data has been discussed only from a point of view corresponding to the singular nature of nucleation. The data shown in Figs. 3 and 5 only represents the final frozen fraction obtained for a defined temperature and electric field strength long times $(t=1.5 \mathrm{~s})$ after applying the impulse voltage, which may be reasonable when only taking into account the long term effect of the impulse voltage. However, nucleation is still a stochastic process governed by molecular motion, regardless of any singular appearance when studied with respect to an appropriate timescale [50-52].
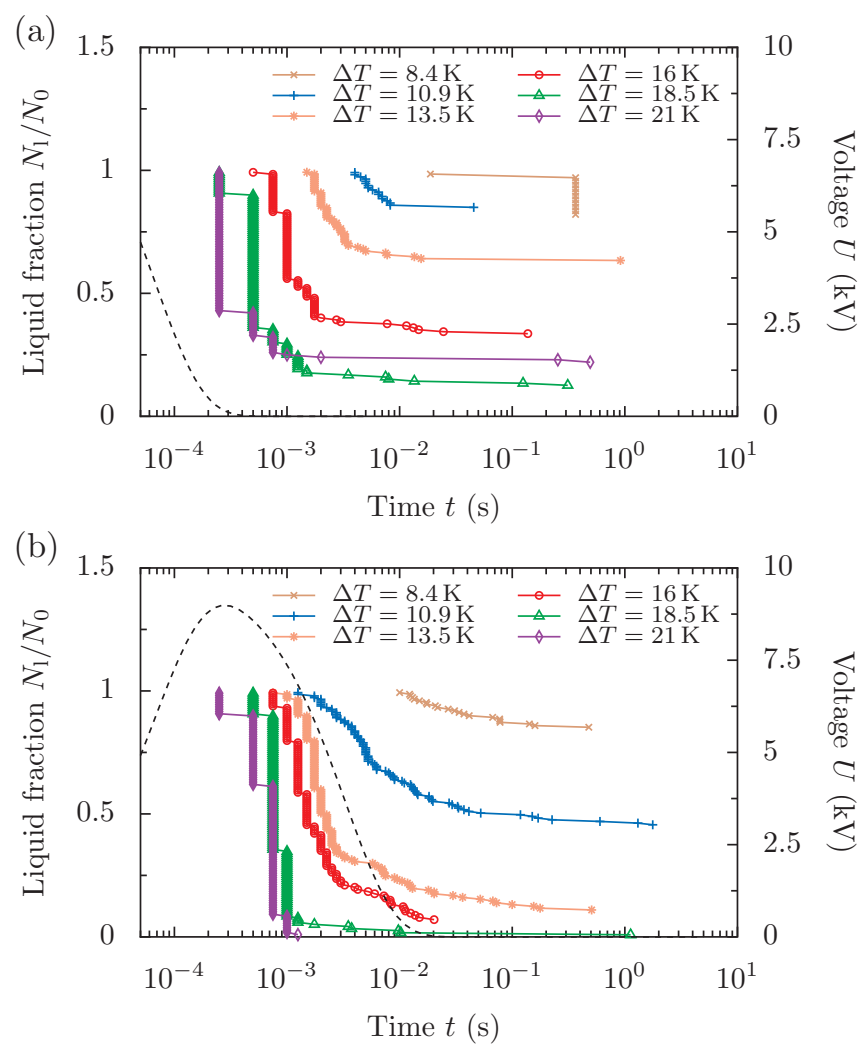

FIG. 8. Liquid fraction $N_{\mathrm{l}} / N_{0}$ depending on time $t$ and supercooling $\Delta T$ under the impact of transient electric fields with $\hat{E}=$ $16.3 \mathrm{kV} / \mathrm{cm}$. Panel (a) shows the resulting liquid fraction for lightning and panel (b) for a switching impulse voltage. Time $t=0 \mathrm{~s}$ refers to the instant when the transient electric field is triggered. The dashed line shows the applied impulse voltage.

The temporal evolution of the liquid fraction of droplets for a constant electric field strength $\hat{E}=16.30 \mathrm{kV} / \mathrm{cm}$ is shown for different ensemble supercooling in Fig. 8. While the data shown in Fig. 8(a) corresponds to a lightning impulse voltage, the data in Fig. 8(b) is obtained from applying a switching impulse voltage. The time $t=0 \mathrm{~s}$ refers to the time instant when the transient electric field is generated. The temporal evolution of the impulse voltage is shown as a dashed line in the figure for both cases. As shown in the figure, for both types of impulse voltage, the droplets do not freeze instantaneously after the impulse voltage is applied, but exhibit significant freezing delays ranging up to almost $\Delta t=1.5 \mathrm{~s}$. Although some droplets may take longer than $\Delta t=1.5 \mathrm{~s}$ to freeze, these are not considered in the present analysis, since at these times no direct influence of the electric field is anymore expected. In fact, droplets with a much longer freezing delay might nucleate due to the stochastic nature of nucleation, which causes droplets to freeze after a delay even without the influence of an electric field. However, this behavior was rarely observed during the experiments.

As shown in Fig. 8, the droplets do not freeze simultaneously, but the time instant of nucleation in the individual droplets covers several orders of magnitude and is clearly stochastically driven. The temporal resolution of the highspeed camera prevents analysis of the nucleation events for 
$t<2.5 \times 10^{-4} \mathrm{~s}$, which all accumulate in the first measurement at $t=2.5 \times 10^{-4} \mathrm{~s}$. Nevertheless, the data shown in Fig. 8 clearly allows examination of the effect of varying exposure time for lightning and switching impulse voltages on the temporal decay of liquid droplets in the ensemble. For lightning impulse voltages and supercooling $\Delta T<21 \mathrm{~K}$, the major onset of nucleation in the droplet ensemble is observed significantly after application of the impulse voltage, when the voltage has already completely decayed. Based on the data for $\Delta T \leqslant 18.5 \mathrm{~K}$, the major onset of nucleation is after field application. However, the limited temporal resolution of the data prohibits a clear conclusion for that supercooling.

In the case of switching impulse voltages, the major onset of nucleation for $\Delta T \geqslant 16 \mathrm{~K}$ is slightly later compared to the situation during a lightning impulse. However, at these later times the actual effect of the switching impulse is much more pronounced compared to the effect of the lightning impulse. For the remaining supercooling the situation concerning the major onset of nucleation is exactly the opposite, i.e., the onset of nucleation is earlier in the case of switching impulse voltages for $\Delta T<16 \mathrm{~K}$. However, the actual effect of the impulse voltage on nucleation is still more pronounced in the case of the switching impulse, also for weaker supercooling. Generally, the very first onset of nucleation observed in the droplet ensemble is significantly spread over approximately two orders of magnitude in the case of the lightning impulse, while it is rather focused to smaller time intervals in the case of the switching impulse, at least for $\Delta T>8.4 \mathrm{~K}$. Moreover, for switching impulse voltages, a clear temporal overlap is observed for $\Delta T>8.4 \mathrm{~K}$ between the onset of nucleation and the field application. For high supercooling the major portion of the nucleation events is observed before the field has completely decayed, but still after the maximum of the applied voltage.

Concluding, the different types of impulse voltage result in a similar temporal evolution of nucleation in a droplet ensemble. However, when considering that the characteristic times of the switching impulse voltage are approximately two orders of magnitude longer than those of the lightning impulse (see Table III), the observed behavior somehow appears to be counterintuitive. For increasing characteristic times of the impulse voltage, the time interval, in which the major part of nucleation takes place, actually decreases. Moreover, at least for $\Delta T<21 \mathrm{~K}$ the major onset of nucleation is rather invariant for the significantly varying characteristic times of the impulse voltages. Therefore, the influence of the impulse voltage on nucleation indeed appears to be based on a rather slow physical process such as fluid flow and resulting shear induced by droplet oscillation. Otherwise, the major onset of nucleation should vary significantly more between the different impulse types. The effect of a switching impulse on droplet deformation is much more pronounced compared to a lightning impulse, and also its effect on ice nucleation is enhanced. While the major onset of nucleation is not significantly changed, the major part of the nucleation events actually takes place over a much smaller time interval for the switching impulse.

Assuming heterogeneous nucleation to occur with a constant rate on the substrate area wetted by a droplet, $A_{\text {wet }}$, the
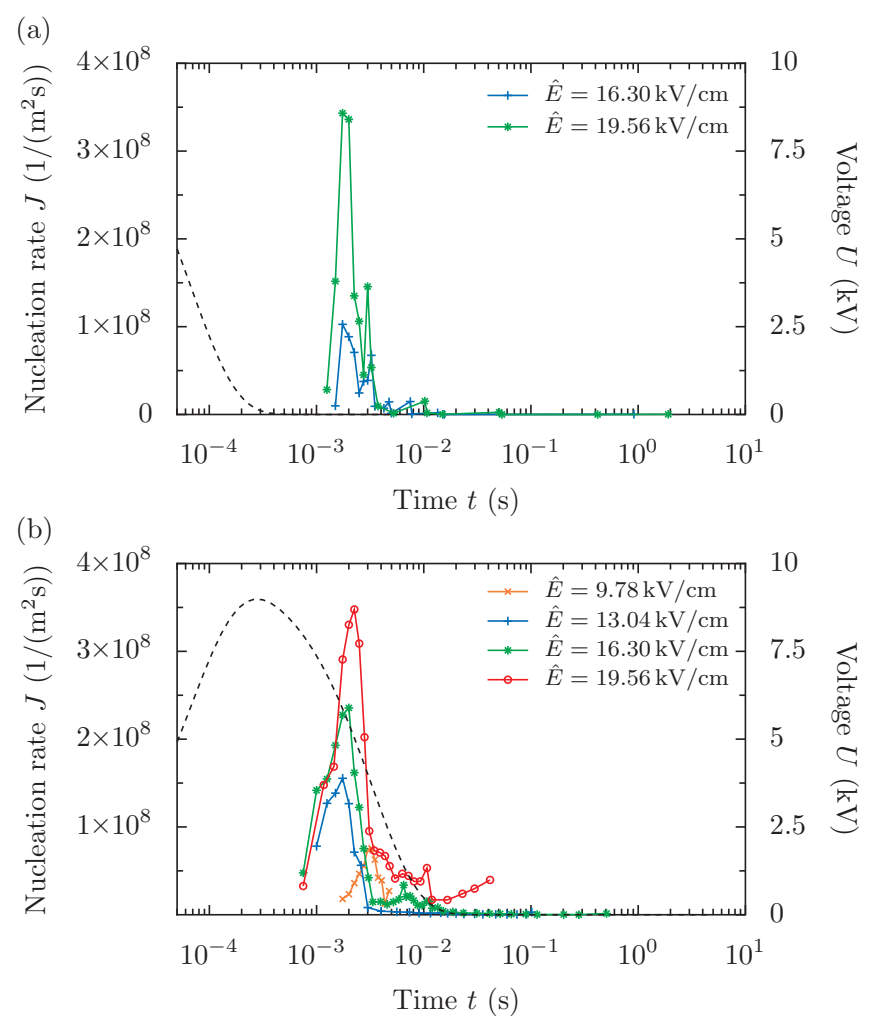

FIG. 9. Temporal evolution of the nucleation rate $J$ depending on the electric field strength $\hat{E}$ at temperature $\vartheta=-13.5^{\circ} \mathrm{C}$, shown for (a) lightning impulse voltage and (b) switching impulse voltage. Time $t=0 \mathrm{~s}$ refers to the instant when the transient electric field is triggered. The dashed line shows the evolution of the applied impulse voltage.

nucleation rate $J(t)$ in dimensions of $\mathrm{s}^{-1} \mathrm{~m}^{-2}$ is related to the fraction of liquid droplets as [50,53]

$$
\frac{N_{\mathrm{l}}(t)}{N_{0}}=\exp \left[-\int_{0}^{t} A_{\mathrm{wet}} J(t) \mathrm{d} t\right] .
$$

The nucleation rate represents the probability of freezing per unit surface area and time, and can be calculated from the experimental data as

$$
J(t)=-\frac{1}{A_{\text {wet }}} \frac{\mathrm{d}\left[\ln \frac{N_{1}(t)}{N_{0}}\right]}{\mathrm{d} t} .
$$

A comparison of the time dependent nucleation rates determined for different electric field strengths using Eq. (2) is shown in Fig. 9 as an example. The data is shown in a semilogarithmic diagram to account for the significant dependence of the nucleation rate on time. Figures 9(a) and 9(b) contain selected data for lightning and switching impulse voltages, respectively, each for a supercooling of $\Delta T=13.5 \mathrm{~K}$. For clarity purposes the data shown in Fig. 9 is reduced compared to the data shown in Fig. 8. Not every nucleation event is shown in the figure but only those data which has been actually used for the calculation of the nucleation rate; i.e., for every video frame only one value of the liquid fraction is shown instead of showing all nucleation events. Moreover, the number of electric field strengths for which data is shown in Fig. 9(a) is reduced because only electric field strength 
$\hat{E} \geqslant 16.30 \mathrm{kV} / \mathrm{cm}$ have a significant impact on ice nucleation at a temperature of $\vartheta=-13.5^{\circ} \mathrm{C}$.

Even though the lightning and switching impulse voltage are characterized through significantly different timescales, the general trend of the time dependent nucleation rate is almost identical for both cases, implying a minor effect of the temporal evolution of the impulse voltage on the temporal evolution of the nucleation rate. Instead, the temporal evolution of the nucleation rate appears to be primarily controlled by mechanisms associated with a significantly longer timescale. After a certain delay with respect to the activation of the impulse voltage, the evolution of the nucleation rate is characterized by a sharp rise until its maximum value is reached after approximately $t \approx 2-4 \mathrm{~ms}$, which is independent of the impulse type, as shown in the figure. Note that the exact time until the maximum nucleation rate is observed depends on the degree of supercooling. The analysis for different supercoolings revealed that a higher supercooling causes the maximum to appear earlier in time. Moreover, the maximum nucleation rate is controlled by the applied electric field strength. The higher the electric field strength the higher is the maximum nucleation rate. While the maximum nucleation rate for an electric field strength of $\hat{E}=19.56 \mathrm{kV} / \mathrm{cm}$ is almost independent of the impulse type, the maximum nucleation rate for lower electric field strength significantly differs for lightning and switching impulses. For the most, the maximum nucleation rate observed for switching impulse voltages is larger compared to the one observed for lightning impulse voltages, confirming the pronounced effect of switching impulse voltages on ice nucleation for the same temperature, as already shown in Fig. 8.

It is noteworthy that the nucleation rate qualitatively has a very similar temporal evolution as the applied voltage. In addition, even though the different impulse types are characterized through significantly varying characteristic timescales, the evolution of the nucleation rate and the instant of appearance of its peak value is very comparable for both types of the electric field. Consequently, the maximum nucleation rate for lightning impulse voltages is observed after the electric field has already completely vanished. In contrast, the maximum nucleation rate for switching impulse voltages is observed while the electric field is still present. Nevertheless, the maximum of the nucleation rate is observed for both impulse types at almost the same moment after field application. This again implies that in the present experiments and for the given electric field strengths, nucleation is significantly affected by a comparably slow physical process such as fluid or droplet motion. This assumption is supported by the fact that the electric history of a droplet ensemble has been shown to have a negligible effect on ice nucleation in a droplet ensemble [27].

The assumption of a relatively slow physical process primarily controlling the temporal evolution of the nucleation rate appears to be confirmed based on the characteristic time of droplet oscillation. The transient electric field causes a transient force acting on the droplet. The resulting force can be assumed to be concentrated at the three-phase contact line, because the electric field strength is significantly increased in this region [54]. Generally, the force acts on the three-phase contact line directed outwards, thus tending to increase the wetted surface area, while the contact line friction counteracts the force of the electric field and damps the droplet response on the transient external force. As reported by Wang and Jones [55] the contact line friction depends on the contact line velocity, so that in the present case a large force is generated because of the small timescale of the impulse. As a result only a small motion of the contact line is recognized and the wetted area is almost not increased. Nevertheless, the disturbance of the shape equilibrium of the droplet results in droplet oscillation which is controlled by the surface tension and the inertia of the droplet. Even for small droplet sizes $\left(d_{\mathrm{d}} \approx 1 \mathrm{~mm}\right)$, viscosity effects can be neglected for the considered system (due to a Laplace number La $=\sigma \rho r_{\mathrm{d}} / \mu^{2} \gg 1$, where $\mu$ is the dynamic viscosity of water, $\sigma$ is the surface tension, $\rho$ is the fluid density and $r_{\mathrm{d}}$ is the radius of the droplet.) In addition, the damping effect through contact line friction is much larger than the effect of viscosity [56]. The characteristic time for droplet oscillation and response in such an arrangement is thus given as [56]

$$
\tau=\left(\frac{\rho r_{\mathrm{d}}^{3}}{\sigma_{\text {oil-wa }}}\right)^{1 / 2}
$$

where $\sigma_{\text {oil-wa }}$ is the surface tension at the oil-water interface. Using $\rho=1000 \mathrm{~kg} / \mathrm{m}^{3}, r_{\mathrm{d}}=0.5 \mathrm{~mm}$ and $\sigma_{\text {oil-wa }}=35.9 \times$ $10^{-3} \mathrm{~N} / \mathrm{m}$ [57] to describe the considered system results in a characteristic time of oscillation of $\tau=1.87 \mathrm{~ms}$. This characteristic time of a droplet, which is mainly affected by surface tension, is in almost perfect agreement with the duration until the maximum nucleation rate is observed after field application, finally indicating droplet motion to be the major pathway through which a transient electric field affects ice nucleation.

As mentioned before, the footprint of the droplet is constant during oscillation, since the three-phase contact line is pinned. Also the fluid particles directly at the wall are at rest, fulfilling the no-slip condition. In contrast, the bulk is in motion as seen in the high-speed videos (compare Fig. 7). This velocity difference leads to a velocity profile inside the droplet which is associated with a certain shear, from which is already known that it may promote ice nucleation [58]. Therefore, it appears evident that the transient electric field indirectly promotes ice nucleation by generating a shear flow inside the droplet. The larger the electric field, the larger is the force acting on the droplet which finally results in a larger droplet oscillation amplitude. The shear inside the droplet also increases for stronger droplet oscillations and thus leads to increased nucleation for higher electric field strengths.

\section{SUMMARY AND CONCLUSION}

Ice nucleation under the impact of transient electric fields has been experimentally examined under well-defined conditions. Two characteristic voltage types, namely standard lightning impulse voltage $(1.2 / 50 \mu \mathrm{s})$ and standard switching impulse voltage $(250 / 2500 \mu$ s), which are typically used in high-voltage engineering and testing, were used to investigate the ice nucleation characteristics in water droplets at varying degrees of supercooling. Numerous nucleation events has been examined by using an ensemble of 40 droplets and by repeating the individual experiments for certain thermal and electrical conditions at least four times. The temporal evolution of the decay of liquid droplets in the ensemble has been 
captured using a high-speed video camera, and ice nucleation has been analysed by considering nucleation as both a singular and a stochastic process.

The experiments reveal a significant effect of transient electric fields on ice nucleation for certain conditions. As reported in previous related studies, the effect of the electric field on ice nucleation depends on the degree of supercooling. For low electric field strengths, $\hat{E} \leqslant 6.52 \mathrm{kV} / \mathrm{cm}$, the electric field does not affect ice nucleation, independent of both the degree of supercooling and the type of the impulse voltage. In contrast, higher electric field strengths may significantly affect ice nucleation, and electric field strengths $\hat{E} \geqslant 19.56 \mathrm{kV} / \mathrm{cm}$ lead to ice nucleation in all droplets of the ensemble for sufficiently high supercooling. In general, ice nucleation is more strongly promoted using standard switching compared to standard lightning impulse voltages for the same electric field strength.

It has been shown that ice nucleation inside the individual droplets does not take place simultaneously. The instant of nucleation in the droplets is rather associated with a significant freezing delay, variable over several orders of magnitude up to several seconds. An analysis of the time dependent nucleation rate reveals that the presence of an electric field increases the nucleation rate. The higher the electric field strength the higher the nucleation rate. Although the temporal resolution of the high-speed camera limits clear conclusions for times $t \leqslant 2.5 \times 10^{-4} \mathrm{~s}$, the experimental data indicates that at a given electric field strength, the major onset of nucleation for higher supercooling is not significantly affected by the type of the impulse voltage, although the characteristic time of the impulse voltage types differs by two orders of magnitude. Therefore, the effect of the impulse voltage on ice nucleation does not appear to be related to fast physical processes such as field induced molecular orientation. Enhanced nucleation through transient impulse voltages is presumably due to macroscopic droplet motion and resulting shear in the resulting flow in the droplet as shown by the comparison of the characteristic timescales of the nucleation rate and the droplet oscillation.

The effect of the standard switching impulse voltage on macroscopic droplet motion is much more pronounced than that of a standard lightning impulse voltage, since it is associated with longer characteristic times commensurate with the droplet oscillations. Consequently, also the effect of the standard switching impulse voltage on ice nucleation is enhanced compared to a standard lightning impulse voltage for the same electric field strength.

In conclusion, strong transient electric fields can be used to actively promote ice nucleation. The performed experiments improve the understanding of the mechanisms during nucleation and indicate that fluid motion plays an important role for ice nucleation in transient electric fields. This is an influence which has not yet been incorporated into nucleation models and presents a challenge for future studies.

\section{ACKNOWLEDGMENTS}

The authors thank the Deutsche Forschungsgemeinschaft (DFG) for financial support of the project within the Collaborative Research Centre SFB-TRR 75, Project No. 84292822.

\section{APPENDIX: SUPPLEMENTAL MATERIAL}

The Supplemental Material [47] contains three videos of the experiments. Selected video showing the impact of a switching impulse $(\hat{E}=18.43 \mathrm{kV} / \mathrm{cm})$ on a single droplet with a volume of $V=50 \mu \mathrm{l}$ at ambient temperature. An exemplary experiment of a droplet ensemble exposed to a lightning impulse voltage generating an electric field strength of $\hat{E}=19.56 \mathrm{kV} / \mathrm{cm}$ at a temperature of $\vartheta=-13.5^{\circ} \mathrm{C}$ and an experiment of a droplet ensemble exposed to a switching impulse voltage generating an electric field strength of $\hat{E}=$ $13.04 \mathrm{kV} / \mathrm{cm}$ at a temperature of $\vartheta=-13.5^{\circ} \mathrm{C}$ [47].
[1] T. Cebeci and F. Kafyeke, Annu. Rev. Fluid Mech. 35, 11 (2003).

[2] L. Makkonen, Cold Reg. Sci. Technol. 14, 163 (1987).

[3] T. Rashid, H. A. Khawaja, and K. Edvardsen, J. Marine Eng. Technol. 15, 79 (2016).

[4] O. Parent and A. Ilinca, Cold Reg. Sci. Technol. 65, 88 (2011).

[5] S. A. Barringer and N. Sumonsiri, Annu. Rev. Food Sci. Technol. 6, 157 (2015).

[6] M. Orlowska, M. Havet, and A. Le-Bail, Food Res. Int. 42, 879 (2009).

[7] Y. You, T. Kang, and S. Jun, Food Eng. Rev. 13, 15 (2021).

[8] M. Farzaneh, Philos. Trans. Roy. Soc. A: Math., Phys. Eng. Sci. 358, 2971 (2000).

[9] M. Farzaneh, Atmospheric Icing of Power Networks, 1st ed. (Springer, Netherlands, 2008).

[10] J. N. Laflamme and G. Périard, Atmos. Res. 46, 99 (1998).

[11] A. Schwaiger, in Elektrische Festigkeitslehre, edited by A. Schwaiger (Springer, Berlin, 1925), pp. 317-440.

[12] K. O. Papailiou and F. Schmuck, Silikon-Verbundisolatoren: Werkstoffe, Dimensionierung, Anwendungen (Springer, Heidelberg, 2012).
[13] M. Farzaneh and J. Kiernicki, IEEE Electr. Insul. Mag. 11, 5 (1995).

[14] M. Farzaneh and W. A. Chisholm, Insulators for Icing and Polluted Environments, IEEE Press series on power engineering (Wiley-Blackwell, Oxford, UK, 2009).

[15] C. Hoose and O. Möhler, Atmos. Chem. Phys. 12, 9817 (2012).

[16] Z. A. Kanji, L. A. Ladino, H. Wex, Y. Boose, M. Burkert-Kohn, D. J. Cziczo, and M. Krämer, Meteorol. Monogr. 58, 1.1 (2017).

[17] C. Marcolli, Sci. Rep. 7, 16634 (2017).

[18] H. R. Pruppacher and J. D. Klett, Microphysics of Clouds and Precipitation, 1st ed., Atmospheric and Oceanographic Sciences Library, Vol. 18 (Springer Science+Business Media B.V, Dordrecht, 2010).

[19] P. V. Acharya and V. Bahadur, Adv. Colloid Interface Sci. 251, 26 (2018).

[20] R. W. Salt, Science 133, 458 (1961).

[21] C. A. Stan, S. K. Y. Tang, K. J. M. Bishop, and G. M. Whitesides, J. Phys. Chem. B 115, 1089 (2011).

[22] S. Wei, X. Xiaobin, Z. Hong, and X. Chuanxiang, Cryobiology 56, 93 (2008). 
[23] Y. Ma, L. Zhong, J. Gao, L. Liu, H. Hu, and Q. Yu, Appl. Phys. Lett. 102, 183701 (2013).

[24] H. R. Pruppacher and M. Neiburger, J. Atmos. Sci. 20, 376 (1963).

[25] H. R. Pruppacher, Pure Appl. Geophys. 104, 623 (1973).

[26] J. B. Doolittle and G. Vali, J. Atmos. Sci. 32, 375 (1975).

[27] J.-M. Löwe, V. Hinrichsen, M. Schremb, and C. Tropea, Phys. Rev. E 103, 012801 (2021).

[28] P. W. Wilson, K. Osterday, and A. D. J. Haymet, Cryo. Lett. 30, 96 (2009).

[29] Svishchev and Kusalik, Phys. Rev. Lett. 73, 975 (1994).

[30] I. M. Svishchev and P. G. Kusalik, J. Am. Chem. Soc. 118, 649 (1996).

[31] J. Y. Yan and G. N. Patey, J. Phys. Chem. A 116, 7057 (2012).

[32] J. Y. Yan, S. D. Overduin, and G. N. Patey, J. Chem. Phys. 141, 074501 (2014).

[33] R. Zangi and A. E. Mark, J. Chem. Phys. 120, 7123 (2004).

[34] G.-X. Nie, Y. Wang, and J.-P. Huang, Front. Phys. 10, 106101 (2015).

[35] F. Yang, R. A. Shaw, C. W. Gurganus, S. K. Chong, and Y. K. Yap, Appl. Phys. Lett. 107, 264101 (2015).

[36] A. Petersen, H. Schneider, G. Rau, and B. Glasmacher, Cryobiology 53, 248 (2006).

[37] A. J. Schwab, Field Theory Concepts (Springer, Berlin, 1988).

[38] A. Küchler, High Voltage Engineering: FundamentalsTechnology-Applications, VDI-Buch (Springer Vieweg, Berlin, 2018).

[39] J.-M. Löwe, M. Schremb, V. Hinrichsen, and C. Tropea, Atmos. Meas. Tech. 14, 223 (2021).

[40] D. M. Harris, T. Liu, and J. W. M. Bush, Exp. Fluids 56, 516 (2015).

[41] T. Storelvmo and I. Tan, Meteorol. Z. 24, 455 (2015).

[42] J.-M. Löwe, V. Hinrichsen, I. V. Roisman, and C. Tropea, Phys. Rev. E 101, 023102 (2020).

[43] G. Vali, Atmos. Meas. Tech. 12, 1219 (2019).

[44] J.-M. Löwe, M. Schremb, V. Hinrichsen, and C. Tropea, in Proceedings of the International Conference on Icing of Aircraft, Engines, and Structures, SAE Technical Paper Series, edited by SAE International (SAE International400 Commonwealth Drive, Warrendale, PA, 2019).

[45] G. Taylor, Proc. Roy. Soc. A: Math., Phys. Eng. Sci. 280, 383 (1964).

[46] J.-M. Löwe, V. Hinrichsen, and C. Tropea, in Proceedings of the 2018 IEEE Electrical Insulation Conference (EIC) (IEEE, Piscataway, NJ, 2018), pp. 443-447.

[47] See Supplemental Material at http://link.aps.org/supplemental/ 10.1103/PhysRevE.104.064801 for videos showing: the impact of a switching impulse $(\hat{E}=18.43 \mathrm{kV} / \mathrm{cm})$ on a single droplet with a volume of $V=50 \mu \mathrm{l}$ at ambient temperature; experiment of a droplet ensemble exposed to a lightning impulse voltage generating an electric field strength of $\hat{E}=19.56 \mathrm{kV} / \mathrm{cm}$ at a temperature of $\vartheta=-13.5^{\circ} \mathrm{C}$; and experiment of a droplet ensemble exposed to a switching impulse voltage generating an electric field strength of $\hat{E}=13.04 \mathrm{kV} / \mathrm{cm}$ at a temperature of $\vartheta=-13.5^{\circ} \mathrm{C}$.

[48] R. T. Collins, J. J. Jones, M. T. Harris, and O. A. Basaran, Nat. Phys. 4, 149 (2007).

[49] M. Shafiei, N. Ojaghlou, S. G. Zamfir, D. Bratko, and A. Luzar, Mol. Phys. 117, 3282 (2019).

[50] G. Vali, Atmos. Chem. Phys. 14, 5271 (2014).

[51] D. Niedermeier, R. A. Shaw, S. Hartmann, H. Wex, T. Clauss, J. Voigtländer, and F. Stratmann, Atmos. Chem. Phys. 11, 8767 (2011).

[52] G. Vali, Atmos. Chem. Phys. 8, 5017 (2008).

[53] P. V. Hobbs, Ice Physics, Oxford classic texts in the physical sciences (Oxford University Press, Oxford, UK, 2010).

[54] M. Vallet, M. Vallade, and B. Berge, Eur. Phys. J. B 11, 583 (1999).

[55] K.-L. Wang and T. B. Jones, Langmuir 21, 4211 (2005).

[56] S. Dash, N. Kumari, and S. V. Garimella, J. Micromech. Microeng. 22, 075004 (2012).

[57] F. Peters and D. Arabali, Colloids Surfaces A: Physicochem. Eng. Aspects 426, 1 (2013).

[58] D. Reguera and J. M. Rubi, J. Chem. Phys. 119, 9888 (2003). 